\title{
The role of PET-CT in radiotherapy planning of solid tumours
}

\author{
Stasa Jelercic and Mirjana Rajer \\ Department of Radiotherapy, Institute of Oncology Ljubljana, Ljubljana, Slovenia
}

Radiol Oncol 2015; 49(1): 1-9.

Received 1 April 2013

Accepted 5 May 2013

Correspondence to: Mirjana Rajer, M.D., Ph.D., Department of Radiotherapy, Institute of Oncology, Zaloška 2, 1000 Ljubljana. Phone: +386 15879 617; Fax: +386 15879 400; E-mail: mrajer@onko-i.si

Disclosure: No potential conflicts of interest were disclosed.

Background. PET-CT is becoming more and more important in various aspects of oncology. Until recently it was used mainly as part of diagnostic procedures and for evaluation of treatment results. With development of personalized radiotherapy, volumetric and radiobiological characteristics of individual tumour have become integrated in the multistep radiotherapy (RT) planning process. Standard anatomical imaging used to select and delineate RT target volumes can be enriched by the information on tumour biology gained by PET-CT. In this review we explore the current and possible future role of PET-CT in radiotherapy treatment planning. After general explanation, we assess its role in radiotherapy of those solid tumours for which PET-CT is being used most.

Conclusions. In the nearby future PET-CT will be an integral part of the most radiotherapy treatment planning procedures in an every-day clinical practice. Apart from a clear role in radiation planning of lung cancer, with forthcoming clinical trials, we will get more evidence of the optimal use of PET-CT in radiotherapy planning of other solid tumours.

Key words: positron emission therapy; radiotherapy; radiotherapy planning; tumour biology

\section{Introduction}

Cancer treatment has undergone major progress in the past decades. Many previously untreatable malignancies are now-days being successfully cured mostly by the combination of various treatment modalities. Radiotherapy (RT) is almost always part of them. When prescribing radical radiotherapy to the patients we need to achieve two goals; the target volume of the tissue irradiated to highdose must encompass the entire tumour and any microscopic extensions of disease and the dose to the normal tissues should be kept as low as possible to avoid major acute and late complications. To arrive at these goals we have to combine technical improvements and clinical experiences. ${ }^{1}$

The most important technical improvements consist of integration of computed tomography (CT) imaging into treatment planning and introduction of computer controlled multileaf collimator system. They enable development of more efficient techniques for dose delivery, such as 3-dimensional conformal radiotherapy (3D-CRT), intensity-modulated radiotherapy (IMRT) and volumetric arc radiotherapy (VMAT). ${ }^{1,2}$ Combined with innovative in-room image-guidance systems they increase precision and accuracy of radiation delivery. ${ }^{3}$

For target delineation we need accurate tumour assessment. Until recently, morphologic (anatomical) imaging with CT and/or MRI scans, was the only information available in the treatment planning process. ${ }^{1,4,5}$ This type of imaging is unable to describe all tumour characteristics. The progress in nuclear medicine has brought an additional perspective to define the extent and characteristics of the tumour. A new concept of 'biologic imaging' has been coined, which provides metabolic, functional, physiological, genotyping and fenotyping information. ${ }^{2}$

Namely, alongside with the innovations in medical physics and nuclear medicine imaging, there has been a major leap in the understanding of tumour biology. It is now recognized that cancer is not a ho- 
mogeneous ensemble of cancer cells with similar attributes, but that consists of subvolumes with very different radiobiological properties such as hypoxic areas that are known to be highly radio-resistant, regions with uncontrolled cellular proliferation. ${ }^{3}$

\section{The role of PET in tumour assessment and RT treatment planning}

The integration of [18F]-fluorodeoxyglucosepositron emission tomography with computed tomography imaging (18FDG-PET-CT) has become an essential part in the evaluation of various types of malignancies. ${ }^{2,6}$ Its role has been widely accepted and confirmed in the staging process, the evaluation of response to treatment and detection of tumour recurrence. ${ }^{7-11}$ However, the role of PET-CT has been proposed and studied in some other settings, especially in the planning of radiation delivery. $5,7,12,13$

Positron Emission Tomography (PET) scanning has become a paradigm for molecular imaging. ${ }^{6} \mathrm{By}$ administering different radiolabeled substances to the patients, we can identify biological characteristics of their tumours non-invasively. ${ }^{1}$ Examples include the uptake of radiolabeled misonidazole as a surrogate for some forms of hypoxia, thymidine for cell proliferation, acetate and choline for lipid metabolism, methionine for amino acid uptake and the most used and studied [F18]-luorodeoxyglucose (FDG) ${ }^{14}$ It is well known that many malignancies have higher metabolism and consequently uptake FDG more than surrounding normal tissues. This allows FDG-PET to image them. ${ }^{1}$ Today more than $95 \%$ of the molecular imaging procedures in oncology make use of FDG. ${ }^{15}$

In radiotherapy planning, biologic imaging is particularly useful when the patient has poorly defined target volumes, (e.g. brain tumour or lung cancer), or when the intent of RT is to deliver higher than standard doses of radiotherapy (called dose escalation) to the tumour in order to kill as many tumour cells as possible and damage as few as possible normal tissue cells (e.g. head and neck cancer, lung carcinoma, prostate carcinoma). ${ }^{16}$ This type of RT planning needs accurate definition of the metabolically active tumour volume and its differentiation from surrounding tissue.

In the International Atomic Energy Agency report 2006-2007 experts concluded that RT based on PET-CT can be more accurate compared to RT based on standard CT in these cases: ${ }^{1}$
- Imaging of lesions not apparent on CT or MRI, such as unsuspected lymph nodes or distant metastases

- Prevention of irradiation of tissues that don't contain tumour cells, such as atelectasis in the case of lung carcinoma

- When chemotherapy is added to RT, response to it can be assessed better with PET$\mathrm{CT}$, than with $\mathrm{CT}$ alone

- Development of "response adapted therapy" in which changes to target volumes could potentially be made during a treatment course.

Additionally, PET-CT is being studied as a replacement of conventional imaging techniques, especially in IMRT planning, which allows the delivery of non-uniform radiation intensity and nonhomogeneous dose distribution inside the target volume. ${ }^{17-19}$ Imaging of biologically diverse tumour sub-volumes could potentially allow administration of different radiation doses to different tumour regions based on suspected tumour burden or radiosensitivity of the region of interest. ${ }^{17}$ For this focal dose escalation (inside the target) with the intent to improve the local control the terms 'dose painting' (2D) or 'dose sculpting' (3D) have been coined. ${ }^{6}$

In conformal 3D radiotherapy planning different volumes (anatomical units) need to be defined in order to deliver RT: gross tumour volume (GTV) includes macroscopically visible tumour and involved lymph nodes; clinical target volume (CTV) is derived from GTV by adding margins to it and includes all subclinical disease; and planning target organ (PTV) which is derived from variations in the size/position of CTV (physiological processes, tumour reduction or swelling) and the patient (weight loss, physiological processes, movements, daily set-up or technical errors). ${ }^{17}$ With development of biologically orientated RT, beside the search for a reliable delineation of the whole malignant lesion, the definition of biologically tumour subvolumes (BTV) is becoming another point of interest. ${ }^{16}$ The idea is that by selectively boosting radio-resistant areas while decreasing the doze to more susceptible zones, local tumour control rates could increase without increased side effects. ${ }^{21}$

When PET or PET-CT is used for RT planning, precise protocols should be followed and consistently applied. ${ }^{22}$ Main uncertainty in applying these protocols in every day clinical practice is tumour contouring based on PET (PET-CT). Up to now PET-based tumour contouring was mainly affected 
by the (investigator's) choice of threshold. There have been some attempts to standardize lesion delineation (GTV) in FDG-PET-derived images: from arbitrary appointed threshold value as a percentage of the maximum uptake (e.g. $40 \%, 50 \%)$, threshold depending on the background signal, to defined absolute standardized uptake value (SUV) (e.g. $2 \pm 0.4)$, and, the most commonly used, the visual interpretation of the PET scan. ${ }^{23-25}$ Nestle et al. compared different techniques of tumour contour definition by FDG-PET that lead to substantially different volumes, especially in patients with inhomogeneous tumors. ${ }^{26}$ Furthermore, Yu et al. tried to determine the cut-off value of SUV by matching the pathologic gross tumour volume from wholemount serial section of stage I non-small cell lung cancer (NSCLC) to PET-based GTV. ${ }^{27}$ In this prospective study, the most appropriate threshold for tumour contour was a 3.0 absolute SUV or $31 \%$ of maximal SUV. In contrast, Devic et al. questioned use of various automatic delineation methods for several reasons: poor resolution of functional imaging, tumour motion, and distinct pathologic subtypes. ${ }^{28}$ In exchange, authors considered a possible option of biologic targeting defined by anatomical modalities (CT and MRI) and multiple biological tracers to determine tumour subvolumes with different biological characteristic and different radiation dose to obtain better tumour control.

For more accurate results, innovations in image quality and reconstruction are required in order to determine the most proficient delineation technique.

The following sections provide a review of the clinical application of PET-CT in radiation treatment planning of some common cancers.

\section{Lung cancer}

When available PET-CT should be used to select patients with NSCLC for treatment with radical RT. ${ }^{1}$ Several studies have shown that inclusion of PET-CT in the staging process of locally advanced NSCLC patients alters the planned treatment in up to $30 \%$ of cases by making them ineligible for radical $\mathrm{RT}$, because of distant metastasis or extensive intrathoracic disease detected by PET-CT..$^{29-31}$ Gregory et al. reported in a prospective study that for patients with NSCLC treated with radical intent, PET-CT-based staging was significantly more powerfully correlated with overall survival than conventional imaging-based staging, across all staging and within cohorts of patients given any particular form of therapy. ${ }^{30}$
In comparison to conventional imaging with CT or PET alone, integrated PET-CT can distinguish malignant lesions from benign lesions with an accuracy of $82 \%$ with varying sensitivity and specificity values (from $79 \%$ to $96 \%$ and from $40 \%$ to $83 \%$, respectively). ${ }^{32}$ For mediastinal node stage, the benefit of PET-CT lies especially in very high negative predictive value over $90 \%$ with the sensitivity, specificity, positive predictive value and accuracy of $73 \%, 80 \%, 78 \%$ and $87 \%$, respectively. ${ }^{33}$

Evaluation of the neoadjuvant chemo-radiotherapy response before potential surgery is another possible implication of functional imaging. ${ }^{34-36}$ The survival of patients with persistent FDG uptake after radiotherapy or surgery is significantly worse than those without residual activity. ${ }^{37} \mathrm{~A}$ recent study published by Usmanij et al. revealed that the degree of early metabolic change already after the second week of chemo-radiotherapy can predict the response to treatment. ${ }^{38}$

Main limitation of FDG-PET as re-assessment tool is that it is not as good as in primary staging of mediastinal lymph nodes (sensitivity and specificity for detection being $63 \%$ and $85 \%$, respectively). Currently it is not recommended as the only diagnostic tool in therapeutic decisions when restaging patients after induction therapy in stage III NSCLC. ${ }^{34}$

Aerts et al. disclosed that areas with residual FDG uptake after high dose (chemo)-radiotherapy largely overlapped with the areas of high FDG uptake locations before treatment..$^{39}$ However, future trials should provide the basis to test if FDG uptake reflects 'radioresistance', by boosting high FDG uptake areas. Moreover, more specific tracer as 18F-fluoromisomidazole may be useful for dosepainting within the tumour as well. ${ }^{40}$

\section{PET-CT in the planning of lung tumour radiotherapy}

Of all the common cancers, lung cancer has been most intensively studied by integrating dual imaging into the radiation treatment management of patients. ${ }^{1,41}$ There are two main reasons to use PET-CT in definitions of the volume needed to be irradiated:

- PET-CT significantly changes lymph node staging in the thorax, usually by showing more positive lymph nodes than CT

- In cases with atelectasis, PET-CT helps to define the border between tumour and atelectasis, allowing a smaller volume of lung to be treated. 


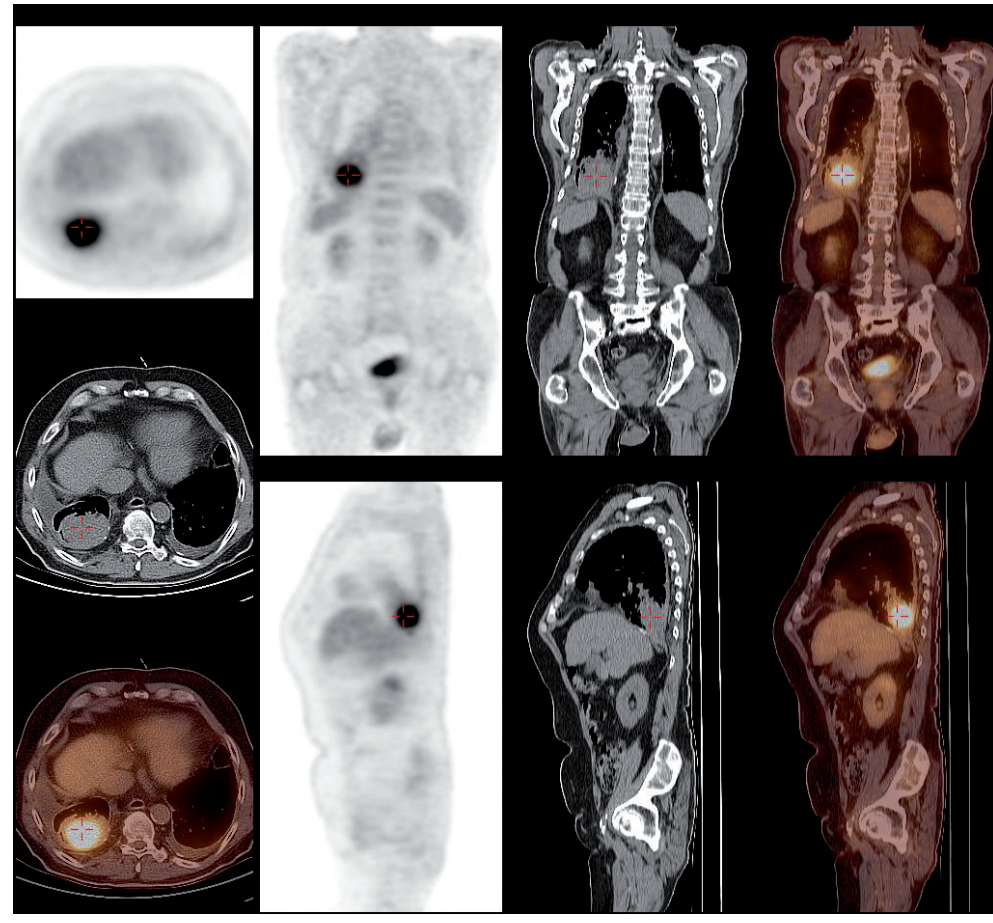

FIGURE 1. Differences in lung tumour and atelectasis seen on PET-CT.

Differences between tumour and atelectasis on PET-CT image are shown in Figure 1.

Nestle et al. have reviewed the results of 18 trials involving 661 patients with lung cancer that compared delineated target volumes, GTV, CTV and PTV using CT alone with the target volumes delineated using additional FDG-PET. ${ }^{41}$ Although the method of comparison was very different between trials, all of them came to the same conclusion that addition of FDG-PET would add essential information to $\mathrm{CT}$ result with significant consequences on GTV, CTV and PTV delineation. The main reasons for size modification lied in the better diagnosis of lymph node involvement and distinguishing tumour from atelectasis. ${ }^{42}$ Another important aspect of integrated PET-CT imaging in radiation planning was reduced interobserver delineation variability in respect to $\mathrm{CT}$ planning alone. The largest reduction was seen in the atelectasis region. ${ }^{43,44}$

\section{Head and neck cancer}

Use of PET-CT can influence the treatment strategy of head and neck cancer patients in various ways. The greatest impact usually results from changes of nodal status before therapy, detection of distant metastasis and/or treatment evaluation. ${ }^{4,45-47}$
In patients with high risk factors for higher nodal stage or distant metastases present (poor differentiation of primary tumour, advanced T stage (T3/ T4), advanced $\mathrm{N}$ stage $(\mathrm{N}>2)$, tumours arising in hypopharynx and larynx, tumours with nodes in lower neck levels $(\mathrm{IV} / \mathrm{IVb}))^{48}$, the addition of pretreatment PET imaging should be routinely used to avoid their over-treatment. ${ }^{49}$ Combined PET-CT showed the highest sensitivity in detecting distant metastases in comparison to only FDG-PET and CT imaging (63\% vs. $53 \%$ and $37 \%) .{ }^{50}$

PET may also be of help in searching for the index tumour in patients presented with lymph node metastases of squamous cell carcinoma from unknown primary to the neck. ${ }^{51}$ Although detection rate of primary tumour ranges from $24 \%-44 \%$ as reviewed by Strojan et al..$^{52}$, PET-CT should be performed when no primary lesion is suggested after thorough physical examination, indirect laryngoscopy and CT/MRI (Figure 2). ${ }^{51}$

The assessment of residual disease in the neck by PET-CT after chemoradiotherapy has become a standard for recognizing patients, who may avoid unnecessary neck dissection. ${ }^{53}$ When evaluation is performed 3 months after the end of chemoradiation, PET-CT exhibit very high negative predictive value $(97-100 \% \text {, as reviewed by Hamoir et al. })^{53}$, and metabolic complete response is predictive for disease- free and overall survival. ${ }^{54}$

\section{PET-CT in the planning of head and neck tumour radiotherapy}

Recent studies have mainly focused on the feasibility of integrating FDG-PET with radiotherapy planning CT with the goal of enhancing tumour localization for IMRT, so the tumour coverage and normal tissue sparing can be optimized..$^{55}$ This is an important issue when very high doses of $70 \mathrm{~Gy}$ or more are being administered to lesions close to radiosensitive vital structures (e.g. brainstem or optic chiasm). ${ }^{1}$ Schwartz et al. examined 20 head and neck cancer patients and studied the potential impact of PET-CT imaging on more tailored IMRT plans, with the exclusion of prophylactic irradiation of PET-negative regions. ${ }^{55}$ Their PET-CT-based IMRT planning did not suffer a geographical nodal miss when correlated with pathological examination, and dose escalation up to 75 Gy was feasible in a selected group of patients without exceeding limiting doses of critical organs.

Several published studies on head and neck cancer patients that compared RT volumes on PET images with standard CT-based planning volumes, 
observed significant differences in target delineation depending on the imaging technique. ${ }^{1}$ Daisne et al. compared GTVs delineated at CT, MRI and PET-CT images with resected tumour specimen. ${ }^{56}$ Although none of these imaging modalities was $100 \%$ accurate, the GTVs delineated at FDG-PETCT were by far the closest to the reference volume from the surgical specimens. Burri et al. tried to correlate the SUV to pathologic specimen size and found that fixed threshold of $40 \%$ of maximum SUV would offer the best compromise between accuracy and the risk of underestimating tumour extent. $^{47}$

Several investigators analyzed the correlation between pre-therapeutic SUV and disease outcome and were reviewed by Inokuchi et al. ${ }^{57}$ They disclosed that not only primary tumour SUVmax, but also SUVmax of cervical lymph nodes is a prognostic factor for local control, disease-free and overall survival. ${ }^{57}$ Greven et al. reported that the changes in primary tumour SUV during and soon after completed treatment were also highly predictive of tumour recurrence compared to CT imaging alone. ${ }^{58}$ They concluded that adaptive therapy planning based on PET-CT may be needed in order to improve the results of the radiation therapy.

As already said, FDG uptake correlates with outcome in head and neck cancer patients and most of loco-regional recurrences occur within FDG-avid areas $^{59}$, which would represent a reasonable target for focal dose escalation..$^{23,24,58-60}$ The concept of dose painting of tumour subvolumes has been evaluated with two different methods: as dose painting by biologic image-defined contours ${ }^{24}$, and as dose painting by numbers, i.e. prescribing dose to points in the target depending of biologic signal intensity. ${ }^{61}$ The latter has been demonstrated to allow higher intratumour doses at similar rates of toxicity. In the study of Duprez et al., the median dose was escalated either to 80.9 Gy to the high-dose CTV or to 85.9 Gy to the GTV. ${ }^{61}$ None of the patients in the study required a treatment break and no Grade 4 acute toxicity was observed. However, which biologic characteristics of tumour and which biologic tracers are most relevant for dose painting are to be found out.

Two other promising PET tracers are 18F-fluoromisonidazole (FMISO) and 18F-fluoroazomycin arabinoside (FAZA), which provide quantitative measurements of tissue hypoxia, one of the main factors affecting treatment resistance in head and neck cancer. ${ }^{62}$ Rajendran et al. performed pretreatment FMISO-PET on 73 head and neck cancer patients and found that both

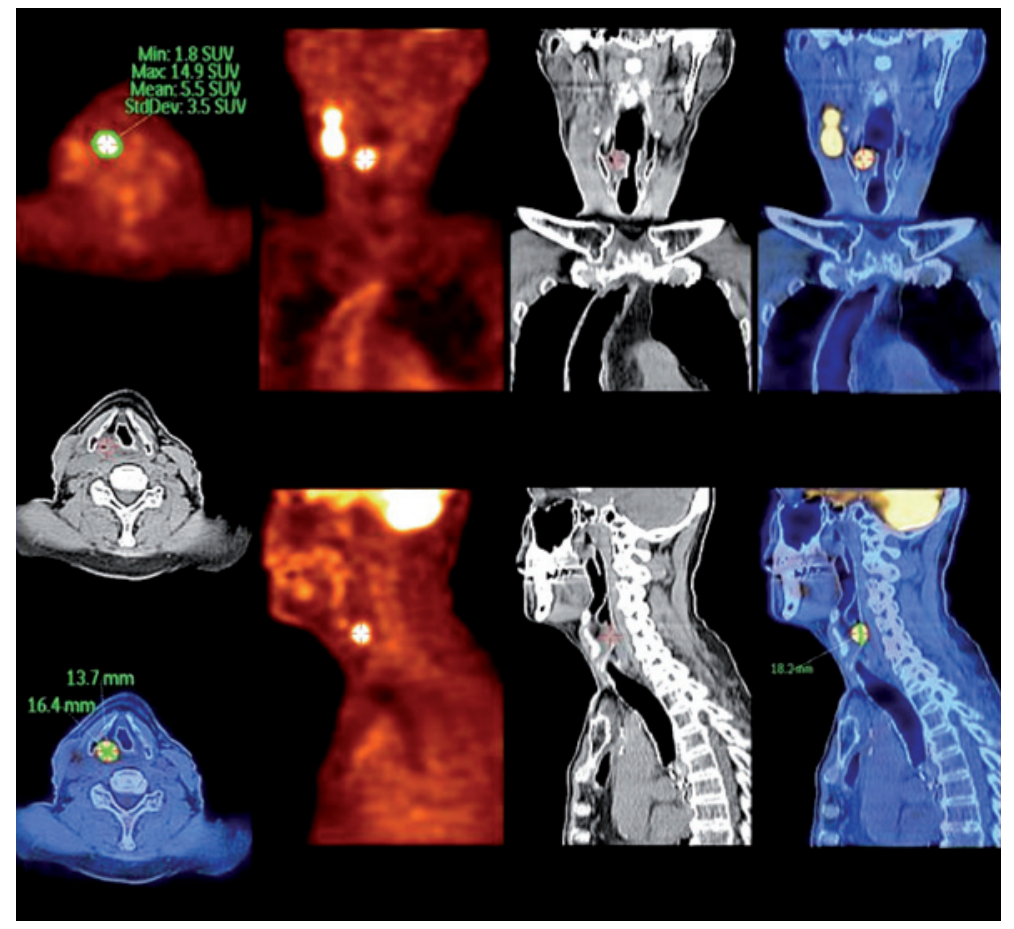

FIGURE 2. PET-CT images of 51-year old male with metastatic squamous cell carcinoma of unknown origin in cervical lymph nodes. The origin was found by PET-CT and later confirmed by a biopsy.

the degree of hypoxia and the size of hypoxic volume measured by FMISO-PET were independent predictive factors of survival. ${ }^{63}$ Mortensen et al. found similar prognostic value of FAZA PET-CT imaging, demonstrating a significant difference in disease free survival rate in patients with non hypoxic tumours and patients with hypoxic tumours (93\% and 60\%, respectively, median follow up 19 months). ${ }^{64}$

Despite the progress in anatomical and functional imaging in defining tumour borders, meticulous, peer reviewed clinical examination cannot be replaced by any of the imaging techniques when determining mucosal extent of primary tumour. ${ }^{65}$ Future large clinical trials are warranted to evaluate the exact position of PET-CT in the radiotherapy treatment of head and neck cancer patients.

\section{Oesophageal cancer}

FDG-PET-CT can provide incremental staging information compared to combined CT and endoscopic ultrasonography in up to $40 \%$ of patients and can change management in one third of patients with oesophageal carcinoma. ${ }^{66}$ In a systematic review by Muijs et al., authors found a sig- 
nificant improvement of the sensitivity (93\%), accuracy (92\%) and negative predictive value (98\%) in the assessment of locoregional lymph nodes by integrated imaging, compared to the use of CT or PET alone. ${ }^{67}$

There is no doubt that addition of FDG- PET in radiotherapy planning has a significant impact on target volume delineation (in $20-94 \%$ of patients), resulting in either reduction or increase in target volumes based on CT images as it is reviewed by Muijs et al. ${ }^{67}$ Trials on patients undergoing resection or endoscopic ultrasound (EUS), which is the gold standard for clinical T-staging, report that PET-based tumour length correlate well with tumour length assessed by pathologic exam or EUS. However, this does not automatically mean correct allocation of malignant tissue in vivo, since the FDG uptake in pathologic areas on one side can be compensated on the other side by inflammation. ${ }^{67}$

Some prospective studies ${ }^{68,69}$ reported about positive contribution of PET-based tumour delineation, which prevented geographic miss by identifying unsuspected malignant involvement in $30-60 \%$ of patients. However, given the poorer sensitivity of FDG-PET for primary tumour, the irradiated volume should not be reduced based on a negative FDG-PET finding in a region with suspect malignant involvement on other diagnostic investigations. $^{70}$

Another method to utilize FDG-PET in RT treatment planning is to reduce inter-and intra-observer variability. Toya et al. showed significantly increased concordance rate in GTV definition (i.e. ratio of the intersection of the GTVs to their union) in inter- and intraobserver comparison, when incorporating FDG-PET-CT images in comparison to CT images of cervical oesophageal carcinoma. ${ }^{71}$ In contrast, Scheuers et al. found no significant effect of the additional use of FDG-PET on the interobserver variability. ${ }^{69}$ Several automatic (or semiautomatic) contouring methods using various thresholds have been tested, but until now have not yielded satisfactory results. ${ }^{67}$

Omloo et al. evaluated the potential prognostic value of FDG uptake in oesophageal cancer patients. ${ }^{72}$ Most of 31 reviewed studies showed that pretreatment FDG uptake and postneoadjuvant treatment FDG uptake, as absolute values, are predictors for survival in univariate analysis. An early decrease in FDG uptake during neoadjuvant therapy is also predictive for pathologic response and survival in most studies. However, for more reliable results, a standardized protocol for FDG-PET acquisition and reconstruction is warranted.

\section{Cervical cancer}

T2 weighted MRI is currently the gold standard for primary tumour staging, especially in determining tumour extension in the parametria (reported accuracy of MRI is 80 to $90 \%$ compared to 60 to $69 \%$ for CT.73,74 In nodal staging PET-CT has been proven as an effective imaging technique in patients with locally advanced cervical carcinoma (i.e. FIGO stage is $\geq \mathrm{IB} 2$ ), with significantly better sensitivity and specificity in comparison to other morphological imaging (PET-CT sensitivity ranging from $77 \%$ to $96 \%$ for pelvic lymph nodes (PELN) and 50\% to $100 \%$ for para-aortic lymph nodes (PALN), specificity from $55 \%$ to $75 \%$ for PELN and $83 \%$ to $95 \%$ for PALN, as reviewed by Magne et al. ${ }^{75}$ In spite a high negative predictive value of $92 \%$ for PALN involvement ${ }^{76}$, many authors still recommend a PALN dissection prior to beginning of chemoradiation, in order to avoid a possible miss of $8 \%$ of positive PALN patients. ${ }^{74}$

Although the presence of metastatic lymph nodes in the PELN and PALN regions does not alter the clinical stage of disease, it alters radiation treatment strategy (either by extending irradiated volume to the common iliac or para-aortic areas or by escalating irradiated dose to the involved lymph nodes).${ }^{74}$ Moreover, Kidd et al. showed that the presence and extent (i.e. the most distant level of involved lymph nodes) of PET positive nodal metastases correlate well with recurrence-free and disease-free survival. ${ }^{77}$

Esthappan et al. attempted to develop a treatment plan to deliver an escalated irradiation dose to involved nodal regions without harming adjacent organ at risk. ${ }^{78}$ By the means of PET-CTguided IMRT they delivered 50.4 Gy to the entire para-aortic region and 59.4 Gy to the positive para-aortic lymph nodes with acceptable irradiating dose to adjacent organs at risk. In their subsequent study, authors provided description of image acquisition, definitions of target volumes based on PET-CT and the doses prescribed to these different target volumes. ${ }^{79}$ Kidd et al. prospectively compared toxicity and clinical outcomes of cervical cancer patients treated with PET-CT-guided IMRT and conventional radiation therapy, treated to the same prescription dose.The late bowel and bladder toxicity (of Grade 3 or more) were present in only $6 \%$ in IMRT group compared to $17 \%$ in non-IMRT group. The IMRT group also showed (unexpectedly) better overall and disease specific survival and a trend towards better recurrence free survival. ${ }^{80}$ 
Several studies demonstrated that post-treatment metabolic response immediately after RT and 3 months after RT are predictive of patient outcome. ${ }^{81,82}$ Schwartz et al. proposed the implementation of routine post-treatment PET-CT, which could affect the approach and timing of salvage therapy of patients with cervical cancer recurrence and would also provide long-term prognostic information only 3 months after completion of therapy. ${ }^{82}$ Yoon et al. found that even earlier assessment (after receiving 54-60 Gy) of metabolic response of PELN in patients with initial PELN metastases correlates to disease free survival rate and could be a useful milestone for selecting patients who need more intense treatment. ${ }^{81}$

\section{Conclusions}

Recent advances in PET-CT can broaden the radiation oncologist's knowledge on the extent of the disease, in order to avoid missing the tumour, with consequent reduction of local control probability, and, on the other hand, avoiding the unjustified irradiation of healthy tissue. Furthermore, the correct definition of the disease stage is mandatory for selection of the most appropriate therapeutic strategy. Another important information for the radiation oncologist relates to biological characteristics of treated tumour, which could affect the response to radiotherapy and are of paramount importance for personalized and biologically oriented radiotherapy. The consensus of using PET-CT information for automated target volume delineation has not been reached yet and is awaiting further validation. PETCT can answer many questions regarding correct disease staging and its biologic characteristics, but its exact role in every-day RT treatment planning is expected to be defined in future clinical trials.

\section{Acknowledgement}

Authors would like to thank doctors Ivana Žagar and Barbara Vidergar-Kralj for the pictures provided.

\section{References}

1. MacManus M, Nestle U, Rosenzweig KE, Carrio I, Messa C, Belohlavek O, et al. Use of PET and PET/CT for radiation therapy planning: IAEA expert report 2006-2007. Radiother Oncol 2009; 91: 85-94.

2. The modern technology of radiation oncology, Volumes 1 \& 2. Van Dyk J, editor. Madison: Medical Physics Publishing Corporation; 2005.
3. Thorwarth D, Geets X, Paiusco M. Physical radiotherapy treatment planning based on functional PET/CT data. Radiother Oncol 2010; 96: 317-24.

4. Vernon MR, Maheshwari M, Schultz CJ, Michel MA, Wong SJ, Campbell $\mathrm{BH}$, et al. Clinical outcomes of patients receiving integrated PET/CT-guided radiotherapy for head and neck carcinoma. Int J Radiat Oncol Biol Phys 2008; 70: 678-84.

5. Petric P, Hudej R, Rogelj P, Blas $M$, Segedin B, Logar HBZ, et al. Comparison of $3 D$ MRI with high sampling efficiency and $2 \mathrm{D}$ multiplanar MRI for contouring in cervix cancer brachytherapy. Radiol Oncol 2012; 46: 242-51.

6. Ling CC, Humm J, Larson S, Amols H, Fuks Z, Leibel S, et al. Towards multidimensional radiotherapy (MD-CRT): biological imaging and biological conformality. Int J Radiat Oncol Biol Phys 2000; 47: 551-60.

7. PET Professional resources and outreach source: 18F-fluorodeoxyglucose (FDG) PET and PET/CT practice guidelines in oncology. A summary of the recommendations and practice guidelines of professional groups. Available April 2013: http://www.snm.org/docs/PET_PROS/ OncologyPracticeGuidelineSummary.pdf

8. Fletcher JW, Djulbegovic B, Soares HP, Siegel BA, Lowe VJ, Lyman GH, et al. Recommendations on the use of 18F-FDG PET in oncology. J Nucl Med 2008; 49: 480-507.

9. Ben-Haim S, Ell P. 18F-FDG PET and PET/CT in the evaluation of cancer treatment response. J NuCl Med 2009; 50: 88-99.

10. Quartuccio N, Treglia G, Salsano M, Mattoli MV, Muoio B, Piccardo A, et al. The role of Fluorine-18-Fluorodeoxyglucose positron emission tomography in staging and restaging of patients with osteosarcoma. Radiol Oncol 2013. 47: 97-102.

11. Kim JS, Jeong YJ, Sohn MH, Jeong HJ, Lim ST, Kim DW, et al. Usefulness of F-18 FDG PET/CT in subcutaneous panniculitis-like T cell lymphoma: disease extent and treatment response evaluation. Radiol Oncol 2012; 46: 279-83.

12. Gregoire V, Haustermans K, Geets X, Roels S, Lonneux M. PET-Based treatment planning in Radiotherapy: A new standard? J NuCl Med 2007; 48: 68-77.

13. Ciernik IF, Dizendorf E, Baumert BG, Reiner B, Burger C, Davis JB, et al. Radiation treatment planning with an integrated positron emission and computer tomography (PET/CT): A feasibility study. Int J Radiat Oncol Biol Phys 2003; 57: 853-63.

14. Haubner R. PET radiopharmaceuticals in radiation treatment planning - synthesis and biological characteristics. Radiat Oncol 2010; 96: 280-7.

15. Halldin C. How to image (tracers). State of the art and future development. [Abstract]. Radiat Oncol 2010; 94(Suppl 1): S5-6.

16. Nestle U. Clinical requirements for target volume selection/ delineation. [Abstract]. Radiother Oncol 2010; 94(Suppl 1): S9.

17. Bentzen SM, Gregoire V. Molecular imaging-based dose painting: a novel paradigm for radiation therapy prescription. Semin Radiat Oncol 2011; 21: 101-10.

18. Madani I, Duthoy W, Derie C, De Gersem W, Boterberg T, Jacobs F, et al. Positron emission tomography-guided, focal-dose escalation using intensitymodulated radiotherapy for head and neck cancer. Int J Radiat Oncol Biol Phys 2007; 68: 126-35.

19. Pinkawa M, Piroth M, Holy R, Klotz J, Nussen S, KrohnT, et al. Intensitymodulated radiotherapy for prostate cancer implementing molecular imaging with 18F-choline PET-CT to define a simultaneous integrated boost. [Abstract]. Radiother Oncol 2010; 94(Suppl 1): S11.

20. Bethesda MD. International Comission on Radiation Units 1993. ICRU Report 50. Prescribing, recording and reporting photon beam therapy.

21. Aerts HJWL, Van Baardwijk AAW, Petit SF, Offermann C, Van Loon J, Houben $\mathrm{R}$, et al. Identification of residual metabolic-active areas within individual NSCLC tumours using a pre-radiotherapy 18Fluorodeoxyglucose-PET-CT scan. Radiother Oncol 2009; 91: 386-92.

22. Sattler B, Lee JA, Lonsdale M, Coche E. PET/CT (and CT) instrumentation, image reconstruction and data transfer for radiotherapy planning. Radiother Oncol 2010; 96: 288-97.

23. Mah K, Caldwell CB, Ung YC, Danjoux CE, Balogh JM, Ganguli SN, et al. The impact of 18FDG-PET on target and critical organs in CT-based treatment planning of patients with poorly defined non-small-cell lung carcinoma: a prospective study. Int J Radiat Oncol Bio Phys 2002; 52: 339-50. 
24. Bradley J, Thorstad WL, Mutic S. Impact of FDG-PET on radiation therapy volume delineation in non-small cell lung cancer. Int J Radiat Oncol Biol Phys 2003; 57: 853-63.

25. Erdi YE, Rosenzweig K, Erdi AK, Macapinlac HA, Hu YC, Braban LE, et al. Radiotherapy treatment planning for patients with non-small cell lung cancer using positron emission tomography (PET). Radiother Oncol 2002; 62: $51-60$.

26. Nestle U, Kremp S, Schaefer-Schuler A, Sebastian-Welsch C, Hellwig D, Rube $C$, et al. Comparison of different methods for delineation of 18FFDG PETpositive tissue for target volume definition in radiotherapy of patients with non-small cell lung cancer. J Nucl Med 2005; 46: 1342-8.

27. Yu J, Li X, Xing L, Mu D, Fu Z, Sun X, et al. Comparison of tumor volumes as determined by pathologic examination and FDG-PET/CT images of nonsmall-cell lung cancer: a pilot study. Int J Radiat Oncol Biol Phys 2009; 75: 1468-74.

28. Devic S, Tomic N, Faria S, Menard S, Lisbona R, Lehnert S. Defining radiotherapy target volumes using 18F-fluoro-deoxy-glucose positron emission tomography/computed tomography: Still a Pandora's box? Int J Radiat Oncol Biol Phys 2010; 78: 1555-62.

29. Deniaud-Alexandre E, Touboul E, Lerouge D, Grahek D, Foulquier JN, Petegnief $Y$, et al. Impact of computed tomography and 18F-deoxyglucose coincidence detection emission tomography image fusion for optimization of conformal radiotherapy in non-small cell lung cancer. Int J Radiat Oncol Biol Phys 2005; 63: 1432-41.

30. Gregory DL, Hicks RJ, Hogg A, Binns DS, Shum PL, Milner A, et al. Effect of $\mathrm{PET} / \mathrm{CT}$ on management of patients with non-small cell lung cancer: results of prospective study with 5-year prospective data. J Nucl Med 2012; 53: 1007-15.

31. Cuaron J, Dunphy M, Rimner A. Role of FDG-PET scans in staging, response assessment and follow-up care for non-small cell lung cancer. Front Oncol 2013; 2: 1-7.

32. Hellwig D, Baum RP, Kirsch CM. FDG-PET, PET/CT and conventional nuclear medicine procedures in the evaluation of lung cancer: A systematic review. Nuklearmedizin 2009; 48: 59-69.

33. De Wever W, Stroobants S, Coolen J, Verschakelen JA. Integrated PET/CT in the staging of non-small cell lung cancer: technical aspects and clinical integration. Eur Respir J 2009; 33: 201-12.

34. Rebollo-Aguirre AC, Ramos-Font C, Villegas Portero R, Cook GJ, Llamas Elvira JM Romero Tabares A. Is FDG-PET suitable for evaluationg neoadjuvant therapy in non-small cell lung cancer? Evidence with systematic review of the literature. J Surg Oncol 2010; 101: 486-94.

35. Cistaro A, Quartuccio N, Mojtahedi A, Fania P, Filosso PL, Ficola U, et al Prediction of 2 years-survival in patients with stage I and II non-small cell lung cancer utilizing 18F-FDG PET/CT SUV quantification. Radiol Oncol 2013; 47: 219-23.

36. Berghmans T, Dusart M, Paesmans M, Hossein-Foucher C, Buvat I, Cataigne $C$, et al. Primary tumor standardised uptake value /SUVmax) measured on fluorodeoxyglucose positron emission tomography (FDG-PET) is of prognostic value for survival in non-small cell lung cancer (NSCLC): a systematic review and meta-analysis (MA) by the European Lung Cancer Working Party for the IASLC Lung Cancer Staging Project. J Thorac Oncol 2008; 3: 6-12.

37. Mac Manus MP, Hicks RJ, Matthew JP, Wirth A, Rischin D, Ball DL. Metabolic (FDG-PET) response after radical radiotherapy/chemoradiotherapy for non-small cell lung cancer correlates with patterns of failure. Lung Cancer 2005; 49: 95-108.

38. Usmanij EA, Geus-Oei LF, Troost EG, Peters-Bax L, van der Heijden EH, Kaanders JH, et al. 18F-FDG PET early response evaluation of locally advanced non-small cell lung cancer treated with concomitant chemoradiotherapy. J Nucl Med 2013; 54: 1528-34.

39. Aerts HJWL, Van Baardwijk AAW , Petit SF, Offermann C, Van Loon J, Houben $R$, et al. Identification of residual metabolic-active areas within individua NSCLC tumours using a pre-radiotherapy 18Fluorodeoxyglucose-PET-CT scan. Radiother Oncol 2009; 91: 386-92.

40. Gagel B, Reinartz P, Demirel C, Kaiser HJ, Zimny M, Piroth M, et al. [18F fluoromisonidazole and [18F] fluorodeoxyglucose positron emission tomography in response evaluation after chemo-/radiotherapy of non-small-cell lung cancer: a feasibility study. BMC Cancer 2006; 6: 51
41. Nestle U, Kremp S, Grosu AL. Practical integration of ${ }^{18}$ F-FDG-PET and PET-CT in the planning of radiotherapy for non-small cell lung cancer (NSCLC): The technical basis, ICRU-target volumes, problems, perspectives. Radiother Oncol 2006; 81: 209-25.

42. De Ruysscher D, Wanders S, van Haren E, Hochstenbag M, Geeraedts W, Utama l, et al. Selective mediastinal node irradiation based on FDG-PET scan data in patients with non-small cell lung cancer: a prospective clinical study. Int J Radiat Oncol Biol Phys 2005; 62: 988-94.

43. Steenbakkers RJ, Duppen JC, Fitton I, Deurloo KE, Zijp L, Comans EF, et al. Reduction of observer variation using matched CT-PET for lung cancer delineation: a three dimensional analysis. Int J Radiat Oncol Biol Phys 2006; 64: 435-48.

44. Ashamalla H, Rafla S, Parikh K, Mokhtar B, Goshwami G, Kambam S, et al. The contribution of integrated PET/CT to the evolving definition of treatment volumes in radiation treatment planning in lung cancer. Int J Radiat Oncol Biol Phys 2005; 63: 1016-23.

45. Lonneux M, Hamoir M, Reychler $H$, Maingon $P$, Duvillard $C$, Calais $G$, et al. Positron emission tomography with [18F]fluorodeoxyglucose improves staging and patient management in patients with head and neck squamous cell carcinoma: a multicenter prospective study. I Clin Oncol 2010; 28: 1190-5.

46. Xu GT, Guan DJ, He ZY. ${ }^{18}$ FDG-PET/CT for detecting distant metastases and second primary cancers in patients with head and neck cancer. A metaanalysis. Oral Oncol 2011; 28: 1190-5.

47. Burri RJ, Rangaswamy B, Kostakoglu L, Hoch B, Genden EM, Som PM, et al. Correlation of positron emission tomography standard uptake value and pathologic specimen size in cancer of the head and neck. Int J Radiat Oncol Biol Phys 2008; 71: 682-8.

48. Haerle SK, Schmid DT, Ahmad N, Hany TF, Stoeckli SJ. The value of (18) F-FDG PET/CT for the detection of distant metastases in high-risk patients with head and neck squamous cell carcinoma. Oral Oncol 2011; 47: 653-9.

49. Ford EC, Herman J, Yorke E, Wahl RL. 18-FDG-PET/CT for Image-guided and Intensity modulated radiotherapy. J Nucl Med 2009; 50: 1655-65.

50. Senft A, De Bree R, Hokstra O, Kuik DJ, Golding R, Oyen WJG, et al. Screening for distant metastases in head and neck cancer patients by chest CT or whole body FDG-PET: a prospective multicenter trial. Radiat Oncol 2008; 87: 221-9.

51. De Bree R. The real additional value of FDG-PET in detecting the occult primary tumour in patients with cervical lymph node metastases of unknown primary tumour. Eur Arch Otorhinolaryngol 2010; 267: 1653-5.

52. Strojan P, Ferlito A, Medina J, Woolgar JA, Rinaldo A, Robbins KT, et al. Contemporary managment of lymph node metastases from an unknown primary to the neck: I. A review of diagnostic approaches. Head Neck 2011; 35: $123-32$.

53. Hamoir M, Ferlito A, Schmitz S, Hanin FX, Thariat J, Weynad B, et al. The role of neck disection in the setting of chemoradiation therapy for head and neck squamous cell carcinoma with advanced neck disease. Oral Oncology 2012; 48: 203-10.

54. Conell CA, Corry J, Milner AD, Hogg A, Hicks RJ, Riscin D, et al. Clinical impact of, and prognostic stratification by, F-18 FDG PET/CT in head and neck mucosal squamous cell carcinoma. Head Neck 2007; 29: 986-95.

55. Scwartz DL, Ford EC, Rajendran J, Yueh B, Coltrera MD, Virgin J, et al. FDG$\mathrm{PET} / \mathrm{CT}$ - guided intensity modulated head and neck radiotherapy: A pilot investigation. Head Neck 2005; 27: 478-87.

56. Daisne JF, Duprez T, Weynand B, Lonneux M, Hamoir M, Reychler H, et al. Tumor volume in pharyngolaryngeal squamous cell carcinoma: Comparison at CT, MR imaging, and FDG PET and validation with surgical specimen. Radiology 2004; 233: 93-100.

57. Inokuchi $\mathrm{H}$, Kodaira $\mathrm{T}$, Tachibana $\mathrm{H}$, Nakamura $\mathrm{T}$, Tomita $\mathrm{N}$, Nakahara $\mathrm{R}$ et al. Clinical usefulness of(18)F Fluoro-2-deoxy-D-Glucose uptake in 178 head-and-neck cancer patients with nodal metastasis treated with definitive chemoradiotherapy: consideration of its prognostic value and ability to provide guidance for optimal selection of patients for planned neck dissection. Int J Radiat Oncol Biol Phys 2011; 79: 747-55.

58. Greven KM, Williams D, Mattern M, West T, Kearns W, Staab D, et al. Prospective study of serial PET/CT imaging for patients with squamous cell cancer of the head and neck and the possibility of adaptive planning. [Abstract]. Int J Radiat Oncol Biol Phys 2009; 75(Suppl 1): S394. 
59. Soto DE, Kessler ML, Piert M, Eisbruch A. Correlation between pretreatment FDG-PET biological target volume and anatomical location of failure after radiation therapy for head and neck cancers. Radioth Oncol 2008; 89: 13-8.

60. Dirix P, Vandecayeve V, De Keyzer F, Stroobants S, Hermans R, Nuyts S. Dose painting in radiotherapy for head and neck squamous cell carcinoma: Value of repeated functional imaging with ${ }^{18} \mathrm{~F}$-FDG PET, ${ }^{18} \mathrm{~F}$-fluoromisonidazole PET, diffusion-weighted MRI, and dynamic contrast-enhanced MRI. J Nucl Med 2009; 50: 1020-7.

61. Duprez F, De Neve W, De Gersem W, Coghe M, Madani I. Adaptive dose painting by numbers for head-and-neck cancer. Int J Radiat Oncol Biol Phys 2011; 80: 1045-55

62. Brizel DM, Sibley GS, Prosnitz LR, Scher RL, Dewhirst MW. Tumor hypoxia adversely affects the prognosis of carcinoma of the head and neck. Int J Radiat Oncol Biol Phys 1997; 38: 285-9.

63. Rajendran JG, Scwartz DL, O'Sullivan J, Peterson LM, Ng P, Scharnhorst J, et al. Tumor hypoxia imaging with F18 fluromisonidazole positron emission tomography in head and neck cancer. Clin Cancer Res 2006; 12: 5435-41.

64. Mortensen LS, Johansen J, Kallehague J, Primdahl H, Busk M, Lassen P, et al. FAZA PET/CT hypoxia imaging in patients with squamous cell carcinoma of head and neck treated with radiotherapy: results from the DAHANCA 24 trial. Radiother Oncol 2012; 105: 14-20.

65. Rosenthal DI, Asper JA, Barker JL, Garden AS, Chao KSC, Morrison WH, et al. Importance of patient examination to clinical quality assurance in head and neck radiation oncology. Head Neck 2006; 28: 967-73.

66. Barber TW, Duong CP, Leong T, Bressel M, Drummond EG, Hicks RJ. 18F-FDG $\mathrm{PET} / \mathrm{CT}$ has a high impact on patient management and provides powerful prognostic stratification in the primary staging of esophageal cancer: A prospective study with mature survival data. J Nucl Med 2012; 53: 864-71.

67. Muijs CT, Beukema JC, Pruim J, Mul VE, Groen H, Plukker JT, et al. A systematic review on the role of FDG-PET/CT in tumour delineation and radiotherapy planning in patients with esophageal cancer. Radiother Oncol 2010; 97: 165-71.

68. Leong $\mathrm{T}$, Everitt $\mathrm{C}$, Yuen $\mathrm{K}$. A prospective study to evaluate the impact of FDG-PET on CT-based radiotherapy treatment planning for esophageal cancer. Radiother Oncol 2006; 78: 254-61.

69. Schreurs LM, Busz DM, Paardekooper GM, Beukema JC, Jager PL, Van der Jagt EJ, et al. Impact of 18-fluorodeoxyglucose positron emission tomography defined target volumes in radiation treatment planning of esophageal cance: reduction in geographic misses with equal inter-observer variabiliy. Dis Esophaus 2010; 23: 483-510.

70. Vrieze O, Haustermans K, DeWever W, Lerut T, Van Cutsem E, Ectors N, et al. Is there a role for FGD-PET in radiotherapy planning in esophageal carcinoma? Radioth Oncol 2004; 73: 269-75.

71. Toya R, Murakami R, Imuta M, Matsuyama T, Saito T, Shiraishi $S$, et al. Impact of Hybrid FDG-PET/CT on Gross Tumor Volume Definition of Cervical Esophageal Cancer: Improving Inter- and Intra-observer Variations. [Abstract]. Int J Radiat Oncol Biol Phys 2011; 81(Suppl 1): S324.

72. Omloo JMT, Van Heijl M, Hoekstra OS, Van Berge Henegouen MI, Van Lanschot JJB, Sloof GW. FDG-PET parameters as prognostic factor in esophageal cancer patients: a review. Ann Surg Oncol 2011; 18: 3338-52.

73. Mitchel DG, Snyder B, Coakley F, Reinhold C, Thomas G, Amendola M, et al. Early invasive cervical cancer: Tumor delineation by magnetic resonance imaging, computed tomography, and clinical examination, verified by pathologic results, in the ACRIN 6651/GIG 183 Intergroup study. J Clin Oncol 2006; 24: 5687-94.

74. Haie-Meder C, Mazeron R, Magne N. Clinical evidence on PET-CT for radiation therapy planning in cervix and endometrial cancer. Radiother Oncol 2010; 96: 351-5.

75. Magne N, Chargari C, Vicenzi L, Gillion N, Messai T, Magne J, et al. New trends in the evaluation and treatment of cervix cancer. The role of FDG-PET. Cancer Treatm Rev 2008; 34: 671-81.

76. Boughanim $M$, Leboulleux S, Rey A, Pham CT, Zafrani $Y$, Duvillard $P$, et al. Histologic results of para-aortic lymphadenectomy in patients treated for stage Ib2/II cervical cancer with negative (18F) Fluorodeoxyglucose positron emission tomography scans in the para-aortic area. J Clin Oncol 2008; 26: 2558-61.

77. Kidd EA, Siegel BA, Dehdashti F, Rader JS, Mutch DG, Powell MA, et al. Lymph node staging by positron emission tomography in cervical cancer: Realtion to prognosis. J Clin Oncol 2010; 28: 2108-13.
78. Esthappan J, Mutic S, Malyapa RS, Grigsby PW, Zoberi I, Dehdashti F, et al. Treatment planning guidelines regarding the use of CT/PET-guided IMRT for cervical carcinoma with positive paraaortic lymph nodes. Int J Radiat Oncol Biol Phys 2004; 58: 1289-97.

79. Esthappan J, Chaudhari S, Santanam L, Mutic S, Olsen J, MacDonald DM, et al. Prospective clinical trial of positron emission tomography/computed tomography image-guided intensity-modulated radiation therapy for cervical carcinoma with positive para-aortic lymph nodes. Int J Radiat Oncol Biol Phys 2008; 72: 1134-9.

80. Kidd EA, Siegel BA, Dehdashti F, Rader JS, Mutic S, Mutch DG, et al. Clinical outcomes of definitive intensity-modulated radiation therapy with fluorodeoxyglucose - positron emission tomography simulation in patient with locally advanced cervical carcinoma. Int J Radiat Oncol Biol Phys 2010; 77: 1085-91.

81. Yoon MS, Ahn SJ, Nah BS, Chung WK, Song HC, Yoo SW, et al. Metabolic response of lymph nodes immediately after RT is related with survival outcome of patients with pelvic node-positive cervical cancer using consecutive $\left({ }^{18} \mathrm{~F}\right)$ fluorodeoxyglucose-positron emission tomography/computed tomography. Int J Radiat Oncol Biol Phys 2012; 84: 491-7.

82. Schwartz JK, Siegel BA, Dehdashti F, Grigsby PW. Association of post-therapy positron emission tomography with tumor response and survival in cervical carcinoma. JAMA 2007; 298: 2289-95. 